\title{
Factores de riesgo e infección del sitio quirúrgico en procedimientos de cirugía ortopédica con prótesis
}

\author{
Rubén Carvajal y Ángela Londoño
}

\section{Risk factors related to surgical site infection in orthopedic prosthesis surgery}

Objective: to identify the risk factors for surgical site infections (SSI) in patients with surgical management fractures with internal fixation or prosthetic material. Materials and Methods: a cross-sectional study was conducted in a tertiary institution in Armenia-Colombia in 2008 and 2009 in 223 patients. Results: The superficial incisional infection frequency was $9,4 \%$ (n: 21$)$, and deep incisional infection was 7,6\% (n: 17); there were no organ/space SSI.; the highest rates of infection were observed in patella fractures $(50 \%)$, acromio-clavicular joint $(25 \%)$, and femur (23,7\%). The infections were caused by: S. aureus, Enterobacter gergoviae y Enterobacter aerogenes. There was an association with clean-contaminated wounds compared to clean wounds (OR 2,2), comminuted fractures $(\mathrm{OR} 2,1)$ multiple fractures (OR 2,9) and patella surgery (OR 3,1). Conclusions: the severity of the fracture and the degree of contamination were predisposing factors to infection.

Key words: Prosthesis-related, infections, surgical wound, infection, handwashing, hospital infections, infection control.

Palabras clave: Infecciones relacionadas con prótesis, infección de herida operatoria, lavado de manos, infección hospitalaria, control de infecciones.

\section{Introducción}

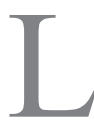

as infecciones asociadas a la atención en salud (IAAS) constituyen un importante problema médico, social y económico, en los países en desarrollo y en los desarrollados, estimándose que ocurren en $8,7 \%$ de los pacientes hospitalizados ${ }^{1,2}$. Entre la IAAS, las infecciones del sitio quirúrgico (ISQs) ocupan el tercer lugar en frecuencia (14 a 17\%) después de la infección urinaria y la neumonía ${ }^{3,4}$; la frecuencia alcanza hasta $73 \%$ de los procedimientos quirúrgicos por lo que su incidencia es uno de los indicadores para evaluar la calidad asistencial $^{5}$. Se ha descrito que entre 13 y $61 \%$ de las ISQs se manifiestan una vez que el paciente ha sido dado de alta del hospital ${ }^{5}$. Las infecciones asociadas a implantes quirúrgicos son generalmente más difíciles de manejar porque requieren largos períodos de antimicrobianos y varios procedimientos quirúrgicos ${ }^{6}$. Las ISQs provocan aumento en la estancia, un incremento de los costos de hospitalización, en los exámenes de diagnóstico, en los controles, en materiales necesarios en el domicilio para la recuperación, etc, y costos indirectos por la incapacidad o trastorno en la vida cotidiana y laboral de los afectados ${ }^{4,7}$.

Los factores de riesgo que determinan la aparición de ISQs son múltiples y pueden estar ligados al paciente, a la intervención y a la técnica quirúrgica utilizada. Factores relacionados con los pacientes son: sitios remotos de infección o colonización, diabetes mellitus, hábito de fumar, uso de corticoesteroides sistémicos, respuesta inmune alterada, desnutrición, obesidad, edades extremas, sexo masculino y estancia hospitalaria prolongada; los factores relacionados con la intervención y la técnica quirúrgica comprenden: el tiempo de la intervención, la antisepsia de piel, la preparación pre-quirúrgica de la piel, la duración del lavado quirúrgico, la preparación del campo quirúrgico, la urgencia de la intervención, el grado de contaminación de la cirugía, un alto nivel ASA, la duración excesiva de la cirugía o de la profilaxis antimicrobiana, la ventilación deficiente de salas de operaciones, métodos de la esterilización, técnica quirúrgica, pobre hemostasia, drenajes, intensidad del trauma tisular y re-intervención ${ }^{3,5,6,8,9}$.

El National Nosocomial Infections Surveillance System (NNISS) utiliza un sistema de riesgo ajustado para pronosticar el riesgo de infección quirúrgica ${ }^{3,7}$ que incluye tres factores de riesgo: el ASA (estado físico del paciente, según la clasificación de la American Society of Anesthesiologists), el grado de contaminación de la herida quirúrgica y la duración del tiempo quirúrgico de la intervención o tiempo $t^{3,7}$. Se han descrito resultados contradictorios al validar el índice de riesgo NNISS; algunos lo han validado por completo mientras que otros lo han ajustado para garantizar su aplicación local ${ }^{10}$.

Este estudio se efectuó con el objetivo de identificar algunos factores de riesgo para ISQ en pacientes con fracturas sometidos a intervención quirúrgica para realizar fijación con material de osteosíntesis o protésico y validar el índice de riesgo NNISS.
Universidad del Quindío, Armenia, Colombia. Facultad de Ciencias de la Salud, Programa de Medicina.

El proyecto fue presentado a convocatoria interna y financiado por la Universidad del Quindío en Armenia-Quindío-Colombia.

Conflictos de interés: Ninguno.

Recibido: 10 de junio de 2011 Aceptado: 3 de mayo de 2012

Correspondencia a:

Ángela Liliana Londoño Franco angelalilianal@uniquindio.edu.co 


\section{Material y Métodos}

Se realizó un estudio de corte transversal en una institución de tercer nivel de la ciudad de Armenia-Colombia, entre 2008 y 2009.

Población de estudio: Cirugías para corrección de fracturas con utilización de material de osteosíntesis o protésico. La muestra se calculó con una confianza del $95 \%$, poder $80 \%$, proporción de exposición en enfermos $47,3 \%$ y OR de $3,0^{3,17}$. Se incluyeron para el análisis 223 procedimientos quirúrgicos (185 sin infección y 38 con ISQ).

Recolección de la información: Se diligenció un protocolo a cada paciente; el seguimiento intra y extrahospitalario fue realizado por traumatólogo y enfermera durante los 12 meses siguientes a la cirugía.

Variables: Se clasificaron las heridas quirúrgicas en: limpia, limpia-contaminada, contaminada y sucia ${ }^{3}$ y las ISQs según los criterios de los Centros de Control de Enfermedades (CDC) de Atlanta: ISQ superficial primaria, ISQ incisional profunda e ISQ de órgano/espacio ${ }^{3,11}$. Para el cálculo del índice de riesgo NNISS ${ }^{3,7}$ se sumó un punto a la puntuación de ASA 3 o superior, heridas quirúrgicas contaminada y sucia, un punto, y tiempo de la intervención superior al tiempo $\mathrm{T}$, un punto; el tiempo $\mathrm{T}$ es el percentil 75 de la duración en minutos calculada para cada intervención realizada en la institución.

Técnicas y procedimientos: Las muestras de pacientes con presunta ISQ fueron recolectadas con técnica estéril, se realizó cultivo para identificación y antibiograma de acuerdo a las recomendaciones de la NCCLS (National Committee for Clinical Laboratory Standards).

Procesamiento y análisis de la información: Se utilizó el paquete estadístico SPSS versión 14. Un primer análisis descriptivo se realizó con el total de la muestra; las variables cualitativas se analizaron mediante la distribución de frecuencias y las cuantitativas con medidas de tendencia central y posición. A través de un análisis bivariado la definición de variables significativas se realizó para las variables cualitativas a través de la distribución de $\chi^{2} \mathrm{y}$ del test exacto de Fisher; para las variables cuantitativas de distribución normal con la t de Student y para las demás con el test de Mann Whitney. Se calculó la odds ratio (OR) y su intervalo de confianza (IC) de $95 \%$ para buscar asociación estadística no causal. El nivel de significación estadística aceptado fue de $5 \%(\mathrm{p}<0,05)$. Se calculó el valor predictor del índice de riesgo NNIS para la variable infección quirúrgica.

Aspectos bioéticos: Se respetaron todas las normas éticas de acuerdo con la Declaración de Helsinki y el Ministerio de Salud de Colombia en la Resolución 8.430 de 1993. Se diligenció de manera individual consentimiento informado.

\section{Resultados}

Se incluyeron 223 procedimientos con un promedio de días de estancia de 6,4 días (IC95\% 5,5-7,3). La localización más frecuente se observó en miembros inferiores; las fracturas se clasificaron como conminutas $32,1 \%$, múltiples $31,6 \%$ y abiertas $19,4 \%$.

Utilización de antimicrobianos profilácticos. Se efectuó profilaxis antimicrobiana en 72,2\% (n: 161/223) de las cirugías. Cefazolina iv fue el fármaco más utilizado, como único medicamento profiláctico en 67,7\% (n: 109/161) de los procedimientos, en dosis de 2 gramos en adultos y $1 \mathrm{gr}$ en niños. En 19,2\% (n: 31/161) se utilizó combinada con otros antimicrobianos (cefazolina más gentamicina 15,5\% (n: 25/161), cefazolina más ciprofloxacina 3,7\% (6/161).

Validación del índice de riesgo NNISS. Se sumó un punto al $13,4 \%$ de los procedimientos en los cuales el puntaje ASA fue de 3 o más. Se clasificaron las heridas quirúrgicas como limpias en $80,3 \%$ (n: 175), limpia contaminada $4,1 \%$ (n: 9), contaminada $14,7 \%$ (n: 32 ) y sucia $0,9 \%$ (n: 2); se sumó un punto al 15,5 (n: 34).

La máxima duración en minutos se observó en los procedimientos realizados sobre fémur (entre 116-118 $\mathrm{min}$ ); en las osteosíntesis de tibia y/o peroné proximal fue de 109 min. En las demás intervenciones de miembros inferiores y superiores el tiempo t varió entre 44 y 90 min como se puede observar en la Tabla 1; en 20,5\% (n: 40) el tiempo $t$ fue superior al calculado por lo cual se sumó a cada uno de estos un punto.

El índice de riesgo NNISS se pudo calcular en 192 procedimientos; en 56,3\% (n: 108) de los procedimientos el índice de riesgo NNISS fue de cero $(0,0)$, en $37,5 \%$ (n: 72) el índice fue de $1,5,7 \%$ (n: 11) tuvieron índice 2 y 0,5\% (n: 1) índice 3.

La frecuencia de ISQ de acuerdo al índice de riesgo NNISS no fue significativa $(\mathrm{p} 0,12)$ aunque se observó un aumento en la frecuencia de infección a medida que aumentaba el puntaje; la frecuencia de ISQ con índice 0 fue de $16,7 \%$ y con índice de $115,3 \%$. De los 11 procedimientos con índice de 2, tuvieron ISQ 27,3\% y sólo hubo un procedimiento con índice 3 y desarrolló ISQ. Se analizó también la relación del índice NNISS con cada tipo de procedimiento quirúrgico sin encontrar relación significativa.

Infección del sitio operatorio y factores de riesgo. Del total de ISQ (38/223) se clasificaron como incisional 
superficial $21(55,3 \%)$ y como ISQ incisional profunda $17(44,7 \%)$; no se presentaron infecciones de órgano/ espacio. Al calcular la frecuencia de acuerdo a la localización, se observó las más elevadas en fracturas de la rótula $(50 \%)$, articulación acromio-clavicular $(25 \%)$, fémur $(18,3 \%)$ y tibia y/o peroné $(17,7 \%)$ (Tabla 2$)$.

En el análisis bivariado se encontró asociación de infección en el sitio operatorio con los siguientes factores de riesgo: ASA II y mayor (OR 2,1), fracturas conminutas (OR 2,1), fracturas múltiples (OR 2,9), y cirugía de rótula $(\mathrm{OR} 3,1)$ (Tabla 3).

Se exploró la relación entre ISQ y los siguientes factores de riesgo sin encontrar diferencias significativas: género $(p=0,4)$, enfermedades de base $(p=0,8)$, anemia $(\mathrm{p}=0,1)$, desnutrición $(\mathrm{p}=0,06)$, cigarrillo $(\mathrm{p}=0,8)$, edad $(p=0,4)$, infecciones distantes al momento del ingreso $(\mathrm{p}=0,9)$, fractura abierta $(\mathrm{p}=0,6)$, intensidad del trauma tisular $(\mathrm{p}=0,3)$, afeitado previo del campo quirúrgico $(\mathrm{p}=0,5)$, antibioterapia intra-operatoria $(\mathrm{p}=0,82)$, tutor externo $(\mathrm{p}=0,09)$, transfusión durante la cirugía $(\mathrm{p}=0,94)$ e ingreso de personas al quirófano durante el procedimiento $(0,62)$.

Así mismo, se exploraron diferencias entre las ISQs superficiales y profundas de acuerdo a todas las variables independientes; se encontraron diferencias no significativas en el estado nutricional donde $40 \%$ de las ISQ profundas se presentaron en presencia de desnutrición mientras que $60 \%$ no la tenían (p 0,06$)$.

Etiología y perfil de susceptibilidad y resistencia de los microorganismos asociados. Del total de ISQs, se pudo cultivar muestras en 15 casos (39,5\%). De ellas, 4 fueron negativas, en 6 se identificó Staphylococcus aureus, en 3 Enterobacter gergoviae y en 2 casos Enterobacter aerogenes + Citrobacter diversus. En todos se detectó resistencia a ampicilina y gentamicina; los bacilos gramnegativos identificados fueron resistentes también a levofloxacina y en uno de los cultivos se observó resistencia a ertapenem y aztreonam.

\section{Discusión}

Entre 5 y $10 \%$ de los pacientes hospitalizados en los países desarrollados contraen una o más infecciones, mientras que en los países en desarrollo, el riesgo de contraer IAAS es entre 2 y 20 veces superior, llegando incluso en algunos a afectar a $25 \%$ de esta población; en las unidades de cuidados intensivos afectan aproximadamente a $30 \%$ de los pacientes y la mortalidad atribuible puede llegar a $44 \%{ }^{2}$. Las ISQs constituyen el segundo tipo de infección hospitalaria y representan aproximadamente $14 \%$ de las mismas en los países desarrollados; en las naciones en desarrollo se informan frecuencias desde 12 a $19 \%$. Se estima que cada año fallecen por esta razón en el mundo al menos entre 2 y $5 \%$ de los 27 millones de personas sometidas a intervenciones quirúrgicas ${ }^{2,3,12}$.

En el presente estudio, la frecuencia de ISQs superficiales $(55,3 \%$ del total) y profundas $(44,7 \%)$ se distribuyó de manera similar a otros estudios que han informado frecuencias de 44,7 y $43,7 \%$, respectivamente ${ }^{13}$ y no se observaron ISQs de órgano-espacio; cerca de $60 \%$ de las infecciones se presentó en procedimientos sobre fémur, tibia y peroné, pero las frecuencias más elevadas se observaron en las cirugías de rótula, articulación

Tabla 1. Duración en minutos de la intervención quirúrgica en minutos y tiempo t (percentil 75)

\begin{tabular}{|llcccc}
\hline Localización & Procedimiento & Rango & Promedio (IC95\%) & Tiempo t \\
Miembros inferiores & Reemplazo de cadera & $45-210$ & 89 & $(69-109)$ & 116 \\
& Fémur: diáfisis y distal & $45-210$ & 93 & $(76-110)$ & 118 \\
& Rótula & $45-105$ & 70 & $(27-112)$ & 97,5 \\
& Tibia y peroné proximal & $50-125$ & 78,8 & $(55-102)$ & 109 \\
& Cuello pie & $30-115$ & 71,7 & $(51-91)$ & 92,5 \\
Miembros superiores & Hombro & $30-90$ & 57,5 & $(6,5-108)$ & 87,5 \\
& Supra-condílea de húmero & & 46,7 & $(29-64)$ & 65 \\
& Otras de húmero & $4-150$ & 63 & $(44-82)$ & 90 \\
& Antebrazo & $15-150$ & 63,6 & $(49,8-77)$ & 90 \\
& Mano & $20-60$ & 34,6 & $(27-42)$ & 44,5 \\
Múltiples fracturas & & $35-160$ & 97 & $(67-127)$ & 131 \\
\hline
\end{tabular}

Tabla 2. Frecuencia de infección en el sitio quirúrgico (ISQ) según localización

\begin{tabular}{|c|c|c|c|c|c|}
\hline Localización & & $(\%)$ & $\begin{array}{c}\text { ISQ superficial } \\
\text { n (\%) }\end{array}$ & $\begin{array}{c}\text { ISQ profunda } \\
\text { n (\%) }\end{array}$ & $\begin{array}{c}\text { Total ISQ } \\
\text { n (\%) }\end{array}$ \\
\hline Fémur & 60 & $(26,9)$ & $4(36,4)$ & $7(63,3)$ & $11(18,3)$ \\
\hline Rótula & 6 & $(2,7)$ & $0 \quad(0,0)$ & $3(50,0)$ & $3(50,0)$ \\
\hline Tibia y/o peroné & 62 & $(27,8)$ & $7 \quad(63,3)$ & $4(36,4)$ & $11(17,7)$ \\
\hline Articulación acromio- clavicular & 4 & $(1,8)$ & $1(100,0)$ & 0 & $1(25,0)$ \\
\hline Húmero & 28 & $(12,6)$ & $2(50,0)$ & $2(50,0)$ & $4(14,3)$ \\
\hline Cúbito y/o radio & 37 & $(16,6)$ & $3(100,0)$ & 0 & $3(8,1)$ \\
\hline Mano & 13 & $(5,8)$ & 0 & 0 & 0 \\
\hline Múltiples & 11 & $(4,5)$ & $4(80,0)$ & $1(20,0)$ & $5(45,5)$ \\
\hline Total & 223 & $(100)$ & $21 \quad(55,3)$ & $17(44,7)$ & $38(100)$ \\
\hline
\end{tabular}

Tabla 3. Factores de riesgo asociados a infección del sitio quirúrgico

\begin{tabular}{|lccc|}
\hline Factor de riesgo (si/no) & Prevalencias a $\%$ & OR (IC95\%) & $\chi^{2}$ (p) \\
ASA II y + vs ASA I & $24,4 / 12,7$ & $2,2(1,1-4,6)$ & $4,8(0,03)$ \\
Fractura : conminuta vs simple & $26,1 / 12,3$ & $2,1(1,2-3,8)$ & $6,4(0,01)$ \\
Fracturas múltiples vs única & $45,5 / 15,6$ & $2,9(1,4-6,0)$ & $6,6(0,01)$ \\
Rótula vs otras localizaciones & $50,0 / 16,1$ & $3,1(1,37,3)$ & $4,7(0,03)$ \\
\hline aPrevalencia de ISQ en cada valor del factor de riesgo analizado. & \\
\hline
\end{tabular}


acromio-clavicular, fémur, tibia y peroné; las más bajas se observaron en cirugías de antebrazo y reemplazos de cadera.

Los elementos utilizados para las osteosíntesis están hechos generalmente de acero inoxidable, titanio o elementos bio-degradables; los procedimientos abiertos con reducción quirúrgica o métodos externos con reducción, estabilización y uso de fijadores externos se asocian, en mayor o menor medida, a un riesgo mayor de infección cuando se comparan con reducciones cerradas sin utilización de los mismos ${ }^{14}$.

Han sido descritos muchos factores de riesgo asociados a las ISQs; entre otras la obesidad, el tabaquismo, medicamentos inmunosupresore ${ }^{15}$, la profilaxis antimicrobiana inadecuada, preparación incorrecta de la piel, duración de la intervención, mala asepsia quirúrgica, tipo de herida quirúrgica y cuidado inapropiado de la misma; problemas nutricionales, diabetes mellitus, inmunosupresión y administración peri-operatoria de derivados sanguíneos ${ }^{2,3}$. En los países en desarrollo se relacionan también con deficiencias de las instalaciones y limitaciones en los suministros de fármacos y otros bienes esenciales ${ }^{2}$.

En nuestro estudio se identificaron como factores de riesgo para ISQ, las fracturas conminutas, las fracturas de huesos múltiples y los procedimientos en rodilla y el ASA mayor a 1; ninguno de los demás factores explorados se encontró significativo en el desarrollo de la infección. Así mismo, se halló una frecuencia significativamente mayor de infecciones profundas en pacientes desnutridos. El estado nutricional es un determinante en la resistencia de los pacientes a la infección ${ }^{12}$. La desnutrición proteicocalórica se ha identificado además como de mayor riesgo para las ISQs más graves ${ }^{12}$. Los estudios coinciden en el mayor riesgo de las fracturas conminutas y del grado de contaminación de la herida quirúrgica como factores de riesgo para $\mathrm{ISQ}^{6,10}$.

La profilaxis antimicrobiana en la hora anterior a la incisión es una medida de eficacia demostrada en la prevención de la ISQ; sin embargo, la OMS refiere que los datos muestran que no se cumple de manera adecuada y entre los motivos para ello se mencionan los costos, la falta de recursos, la administración peri-operatoria que se realiza demasiado pronto, demasiado tarde o de forma errática, volviéndola ineficaz para el paciente ${ }^{3,16}$. Las recomendaciones de profilaxis en cirugía reflejan que existe un consenso entre los cirujanos con respecto a la necesidad de administración de antimicrobianos iv con amplio espectro bactericida de acuerdo a los contaminantes que se esperan según el sitio de la intervención; sin embargo, no hay claridad de las indicaciones o el tiempo de utilización; algunos recomiendan dosis única ${ }^{3,17}$ y otros múltiples manteniendo la protección incluso hasta las $72 \mathrm{~h}$ posteriores a la cirugía ${ }^{17}$. Se recomienda que en heridas clasificadas como contaminadas o sucias el antimicrobiano se deba suministrar como terapéutico y no como profiláctico ${ }^{16}$.

En el presente estudio, el antimicrobiano más utilizado fue cefazolina, coincidente con el más recomendado y empleado en la literatura médica ${ }^{10}$; se considera de elección en este tipo de cirugías en particular en heridas limpias y en la mayoría de limpias-contaminadas por su amplio espectro, bajo volumen de distribución $(0,13 \mathrm{~L} /$ $\mathrm{kg})$, elevado pico sérico $(188 \mu \mathrm{g} / \mathrm{ml})$ a los $5 \mathrm{~min}$ de la administración de $1 \mathrm{~g}$ iv, alta vida media $(1,8 \mathrm{~h})$, escasa toxicidad y buena tolerancia ${ }^{3,17}$. En caso de alergia a cefalosporinas y dependiendo de la edad, también se puede utilizar aztreonam; cuando se requiera cobertura contra anaerobios, clindamicina o metronidazol pueden garantizarla ${ }^{3}$.

A pesar de los pocos cultivos positivos, se encontró que los microorganismos asociados y la frecuencia tienen la misma distribución observada en la literatura científica, el primer lugar para $S$. aureus seguido en frecuencia por E. gergoviae, E. aerogenes y C. diversus. En muchas ISQs la principal fuente es la microbiota endógena del paciente ${ }^{3}$ pero $S$. aureus es un frecuente contaminante de heridas quirúrgicas y representa la primera causa de estas infecciones ${ }^{3,18}$; su virulencia se relaciona con la producción de toxinas y enzimas que originan respuesta inflamatoria en los tejidos infectados y su gran capacidad de sobrevivir en el interior de los polimorfonucleares ${ }^{18}$. La transmisión ocurre generalmente por contacto directo; en condiciones experimentales se ha determinado que la infección viral del tracto respiratorio superior se asocia con aumento de la dispersión en el aire de $S$. aureus de portadores nasales, que puede ir hasta una distancia de 4 metros $^{19}$. Las infecciones hospitalarias por S. aureus resistente a meticilina (SARM) se han asociado a larga estancia, elevada mortalidad e incremento en los costos; se encuentran con mayor frecuencia en hombres y mayores de 60 años ${ }^{20}$.

Staphylococcus aureus y las especies de Enterobacter aisladas en este estudio mostraron resistencia a cefotaxima y gentamicina. La resistencia a carbapenems que fue observada en un solo aislado del presente estudio. En Colombia en 2004 se informó antes un porcentaje de resistencia de $S$. aureus a gentamicina de $47 \%{ }^{21}$.

En el presente estudio no se encontró relación entre la ISQ y el puntaje NNISS; sería necesaria una muestra mayor para buscar diferencias ya que hubo muy pocos con puntaje de 3 lo cual no permitió un análisis adecuado. Se han descrito resultados contradictorios al validar el índice de riesgo NNISS; algunos lo han validado por completo mientras que otros lo han ajustado para garantizar su aplicación local ${ }^{10}$. En un estudio realizado en España, se validó el puntaje NNISS para un total de 6.200 cirugías observando que la tasa de infecciones en cirugía limpia fue de $2,2 \%$, la de limpia-contaminada, de $9,1 \%$, la de contaminada, de $11,4 \%$ y la de sucia, $19,1 \%$. 
La frecuencia de ISQ fue en pacientes con ASA I: 4,0\%, ASA II: $8,2 \%$, ASA III: $13,5 \%$, ASA IV: $19,5 \%$ y ASA $\mathrm{V}: 33,3 \%$; con un tiempo intervención menor al percentil 75 ISQ $6,97 \%$, y mayor del percentil 75 de $23,0 \%$. Con índice NNISS $0: 3,9 \%$, NNISS 1: 8,1\%, NNISS 2: $22,0 \%$ y NNISS 3: $37,2 \%{ }^{5}$.

La duración de la intervención es el factor del índice NNISS que más influye en la tasa de infecciones (OR: 3,43 , frente a 2,60 del grado de contaminación y 2,20 del nivel ASA $)^{5,10}$.

A pesar de la influencia de los factores que configuran el índice NNISS, el rango de la tasa de infecciones para cada uno de los índices sigue siendo muy amplio, lo que indica que deben existir otros factores no incluidos en dicho índice. Por ejemplo, la edad media de los pacientes con una ISQ es significativamente más alta que de los pacientes sin infección $(\mathrm{p}<0,01)$, y la tasa de infecciones en cirugía urgente es significativamente más alta que la correspondiente a la cirugía programada $(10,5$ frente a $7,1 \% ; \mathrm{p}<0,001)^{5}$.

En el Hospital Erasmo Meoz de Cúcuta, en 2003 se realizó un cálculo del tiempo quirúrgico promedio en minutos para cirugías de ortopedia describiendo lo siguiente: osteosíntesis de maléolo externo con placa o tornillos: 22 a 24 min; reducción cerrada de fractura de tibia y peroné con tutor externo: $40 \mathrm{~min}$; osteosíntesis de tibia y peroné y de diáfisis de fémur: 50 min; osteosíntesis de húmero-cúbito y radio-falanges-codo-osteosíntesis fractura inter-trocantérea o supracondílea de fémur: $30 \mathrm{~min}$. Se calculó el tiempo T equivalente al percentil 75 para todas las osteosíntesis en $135 \mathrm{~min}^{10}$. En el presente trabajo, el percentil 75 de todas las osteosíntesis fue menor con 95 min y una duración máxima de 210 min. Los mayores tiempos quirúrgicos medidos con el percentil 75 (tiempo t) se observaron en las osteosíntesis de fémur y pierna y de ellas, los procedimientos sobre diáfisis de fémur y en el tercio proximal de tibia y peroné con 150 y 109 min, respectivamente. Todos las demás osteosíntesis tuvieron un tiempo t alrededor de $90 \mathrm{~min}$.

En conclusión, el riesgo de ISQ se relacionó más con la gravedad de las fracturas y la condición general del paciente al momento de la cirugía. No se logró validar el índice de riesgo NNISS por el tamaño de la muestra pero sí se observó como: a mayor puntaje mayor riesgo, y se recomendó a la institución tenerlo en cuenta para la valoración del riesgo de infección.
No es posible erradicar las infecciones nosocomiales, pero se ha comprobado que varias estrategias sencillas y baratas reducen su incidencia. El Reto Mundial por la Seguridad del Paciente 2005-2006: «Una atención limpia es una atención más segura» está centrando parte de su atención en mejorar las normas y prácticas de higiene de las manos en la atención sanitaria y en ayudar a aplicar las intervenciones eficaces ${ }^{16}$. Así mismo, el reto: " $L a$ cirugía segura salva vidas" incluye acciones de seguridad tales como las prácticas inadecuadas de seguridad de la anestesia, las infecciones quirúrgicas evitables o la comunicación deficiente entre los miembros del equipo quirúrgico y se ha definido una serie de controles de seguridad que podrían llevarse a cabo en cualquier quirófano; el resultado ha sido la Lista OMS de verificación de la seguridad de la cirugía, la cual debe adaptarse a las condiciones de cada sitio, de acuerdo a los recursos y necesidades ${ }^{16}$. La "Lista de verificación" es una herramienta sencilla y práctica que puede ser utilizada por cualquier equipo quirúrgico del mundo para garantizar de forma eficiente y rápida la observancia de medidas pre-operatorias, intra-operatorias y post-operatorias que aportan al paciente beneficios demostrados"16.

\section{Resumen}

Objetivo: Identificar factores de riesgo para infecciones del sitio quirúrgico en pacientes con fracturas quirúrgicas de manejo con material de osteosíntesis o prótesis. Material y Método: Se realizó un estudio de corte transversal en una institución de tercer nivel de Armenia- Colombia en 2008 y 2009 en 223 pacientes. Resultados: La frecuencia de infección incisional superficial fue de 9,4\% (n: 21), y de incisional profunda 7,6\% (n: 17); no hubo infecciones de órgano/espacio; las mayores frecuencias de infección se observaron en fracturas de rótula $(50 \%)$, articulación acromio- clavicular (25\%) y fémur $(23,7 \%)$. La etiología infecciosa fue por: Staphylococcus aureus, Enterobacter gergoviae y Enterobacter aerogenes. Se encontró asociación con heridas limpia-contaminadas comparadas con heridas limpias (OR 2,2), fracturas conminutas (OR 2,1), fracturas múltiples (OR 2,9), y cirugía de rótula (OR 3,1). Conclusiones: La gravedad de la fractura y el grado de contaminación se evidenciaron como predisponentes para infección.

\section{Referencias bibliográficas}

1.- Organización Mundial de la Salud. Prevención de las infecciones nosocomiales. Guía práctica. OMS [Internet]; 2003. Disponible en: http:// www.who.int/csr/resources/publications/ ES_WHO_CDS_CSR_EPH_2002_12.pdf (accedido: noviembre de 2009).
2.- World Health Organization. WHO Guidelines on hand hygiene in health care first global patient safety challenge clean care is safer care. World Health Organization 2009. Disponible en: http://whqlibdoc.who.int/ publications/2009/9789241597906_eng.pdf (accedido: julio de 2010).

3.- Centers for Disease Control and Prevention.
Guidelines for prevention of surgical site infection. Infect Control Hosp Epidemiol 1999; 20 (4): 247-77.

4.- Perencevich E N, Sands K E, Cosgrove S E, Guadagnoli E, Meara E, Platt R. Health and economic impact of surgical site infections diagnosed after hospital discharge. Emerg Infect Dis 2003, 9 (2): 196- 203. 
5.- Iñigo JJ, Bermejo B, Oronoz B, Herrera J, Tarifa A, Pérez F, et al. Infección de sitio quirúrgico en un servicio de cirugía general. Análisis de cinco años y valoración del índice National Nosocomial Infection Surveillance (NNIS) index Cir Esp 2006; 79 (4): 224-30.

6.- Darouiche R O. Treatment of infections associated with surgical implants. N Engl J Med 2004; 350: 1422-9.

7.- Kaye K S, Sands K, Donahue J G, Chan K A, Fishman P, Platt R. Preoperative drug dispensing as predictor of surgical site infection. Emerg Infect Dis 2001; 7 (1): 57- 65.

8.- Southwell J P, Russo R R, March L, Cumming R, Cameron I, Brnabic A J. Antibiotic prophylaxis in hip fracture surgery: a metaanalysis. Clin Orthop 2004; (419): 179-84.

9.- Soleto L, Pirard M, Boelaert M, Peredo R, Vargas R, Gianella A, Vander Stuyft P. Incidence of surgical-site infections and the validity of the National Nosocomial Infections Surveillance System risk index in a general surgical ward in Santa Cruz, Bolivia. Infect Control Hosp Epidemiol 2003; 24 (1): 26-30.

10.- Albornoz J C. Fracturas de maléolo externo e tobillo: Tratamiento con osteosíntesis mínima. Revista Venezolana de Cirugía Ortopédica y Traumatología 2003; 35 (1): 7-12.

11.- Horan TC, Andrus M, Dudeck M A.
CDC/NHSN surveillance definition of health care-associated infection and criteria for specific types of infections in the acute care setting. Amer J Infect Control 2008; 36 (5): 309-32.

12.- Sociedad Argentina de Infectología. Documento de consenso: Prevención de infección del sitio quirúrgico y seguridad del paciente en el pre- intra y post quirúrgico. Memorias del VIII Congreso SADI 2009 Junio 11 y 12. Mar del Plata Argentina 2009. Disponible en: http:// www.sadi.org.ar/files/CONSENSOISQFINAL. PDF (accedido: noviembre de 2010).

13.- Vilar D, Sandoval S, Gordillo P, De la Rosa M, Sánchez G, Volkow P. Vigilancia de las infecciones de herida quirúrgica. Experiencia de 18 meses en el Instituto Nacional de Cancerología. Salud Pública Mex 1999; 41 (1): S44-S50.

14.- García C, Ortega D. Elementos de osteosíntesis de uso habitual en fracturas del esqueleto apendicular: evaluación radiológica. Rev Chil Radiol 2005; 11 (2): 58-70.

15.- Morales S, López C, Moreno L, Munévar M, Linares C, Álvarez C. Infección del sitio de la operación en un hospital universitario de tercer nivel. Universitas Médica 2005; 46 (2): 42-7.

16.- Organización Mundial de la Salud. Alianza mundial para la seguridad del paciente. Directrices de la OMS sobre higiene de las manos en la atención sanitaria (borrador avanzado): Resumen unas manos limpias son manos más seguras. OMS 2005.

17.- Calzadilla V, Leyva F, Álvarez J, Castillo I, Reyes A, Almira M. Profilaxis peri operatoria con cefazolina en cirugía ortopédica. Rev Cub Med Mil 2007; 36 (3): 1-6.

18.- Valle G M, Décalo M M, McCook L, Piloto M, Sebazco C, Ruiz V. Modelo de herida infectada. Instituto Superior de Medicina Militar «Dr. Luis Díaz Soto». Rev Cub Med Mil 2005; 34 (2).

19.- Siegel JD, Rhinehart E, Jackson M, Chiarello L, and the Healthcare Infection Control Practices Advisory Committee. Guidelines for isolation precautions: preventing transmission of infectious agents in healthcare settings. CDC 2007. Disponible en: http://www. cdc.gov/hicpac/pdf/isolation/Isolation2007.pdf (accedido: enero de 2011).

20.- Klevens M, Morrison M A, Nadle J, Petit S, Gershman K, Ray S, et al. Invasive methicillinresistant Staphylococcus aureus infections in the United States. JAMA 2007; 298 (15): 1763- 71.

21.- Organización Panamericana de la Salud, USAID. Informe Anual de la Red de Monitoreo/ Vigilancia de la Resistencia a los Antibióticos. 27 al 29 de julio de 2005. Brasilia, Brasil. OPS 2005. Disponible en: http://www.paho.org/ Spanish/AD/DPC/CD/amr-2004.pdf (accedido: enero de 2011). 